\title{
Assessing the Chemical Status of Water from Wells Which Supply Farms Located on Romania's Territory. Part II
}

\author{
Cristina EL MAHDY ${ }^{*}$, Silvana POPESCU ${ }^{2}$, Anca BOARU ${ }^{1}$, Cristin BORDA ${ }^{2}$ \\ ${ }^{1}$ Faculty of Animal Husbandry and Biotechnologies,University of Agricultural Sciences and Veterinary \\ Medicine, Calea Mănăștur street 3-5, Cluj-Napoca, România \\ ${ }^{2}$ Faculty of Veterinary Medicine, University of Agricultural Sciences and Veterinary Medicine, Calea \\ Mănăștur street 3-5, Cluj-Napoca România \\ * corresponding author: cristina.hegedus@usamvcluj.ro
}

Bulletin UASVM Animal Science and Biotechnologies 73(2)/ 2016

Print ISSN 1843-5262; Electronic ISSN 1843-536X

DOI:10.15835/buasvmcn-asb: 12155

\begin{abstract}
The study was performed in 63 wells which belong to the decentralized system from 5 counties from Romania: AB- 7 wells, BZ- 6 wells, CJ-33 wells, MS-1 wells, SJ- 6 wells taking into account chemical parameters: nitrites (SR ISO 6777:1996), nitrates (SR ISO 7890:1-1998), the report nitrates/nitrites in accordance with L. 458/2002. Results reveals values of $\mathrm{NO}_{2}: 0.32 \pm 0.08 \mathrm{mg} / \mathrm{dm}^{3} \mathrm{AB}, 0.23 \pm 0.08 \mathrm{mg} / \mathrm{dm}^{3} \mathrm{BZ}, 0.33 \pm 0.05 \mathrm{mg} / \mathrm{dm}^{3} \mathrm{CJ}, 0.23 \pm 0.03 \mathrm{mg} / \mathrm{dm}^{3} \mathrm{MS}$, $0.09 \pm 0.02 \mathrm{mg} / \mathrm{dm}^{3} \mathrm{SJ}$, and $\mathrm{NO}_{3}: 35.71 \pm 7.53 \mathrm{mg} / \mathrm{dm}^{3} \mathrm{AB}, 48.66 \pm 5.92 \mathrm{mg} / \mathrm{dm}^{3} \mathrm{BZ}, 23.54 \pm 2.82 \mathrm{mg} / \mathrm{dm}^{3} \mathrm{CJ}, 19.63 \pm 4.64$ $\mathrm{mg} / \mathrm{dm}^{3} \mathrm{MS}, 31.16 \pm 5.12 \mathrm{mg} / \mathrm{dm}^{3} \mathrm{SJ}$ which, theoretically include the waters in terms of two parameters in drinking water and good chemical states, but, individual samples in terms of nutrient regime presents exceedance influenced by poor cleaning, hygienisation and disinfection of wells.
\end{abstract}

Keywords: cow, drinking water, $\mathrm{NO}_{2}, \mathrm{NO}_{3}$

\section{INTRODUCTION}

Purpose of this paper was to identify the degree of cleanliness or pollution 63 wells from five counties located in SE, NW-eastern and Romania center: Alba (AB localization: central part of Romania 7 wells), Buzau (BZ- localization: SE 6 wells), Cluj (CJ localization: west central 33 wells), Mures (MS localization: central - northern 11 wells), Salaj (SJ - localization: northwest 6 wells)], used as a source of drinking water for cattle, related by the presence of biogenic elements based on nitrogen $\mathrm{NO}_{2}$ - intermediate form and $\mathrm{NO}_{3}$ - final form. Impurification with nitrates can have telluric nature, by using of fertilizers based on nitrogen (Harty, 2012) or poor sanitation and failure to comply hygiene and sanitary conditions or location of wells (Iacob et al, 2012). Nitrite levels in water which are over 4 ppm may be toxic to cattle (Grant, 1993).

\section{MATERIALS AND METHODS}

In this part, were followed: determination of nitrites (SR ISO 6777:1996), nitrates (SR ISO 7890/1:1998), report $\mathrm{NO}_{3}$ and $\mathrm{NO}_{2}$ in accordance with L. 458/2002. Study was conducted on a number of 63 wells located in the territory of five counties from Romania: $\mathrm{AB}, \mathrm{BZ}, \mathrm{CJ}, \mathrm{MS}, \mathrm{SJ}$, results being compared with allowed limits and those for animals farm requirements, using as statistical tests accepted: non parametric test Krushall-Wallis and comparing the values after a value established (Fisher's Test).

\section{RESULTS AND DISCUSSION}

By point of view of $\mathrm{NO}_{2}$ parameter, 48 (76.19\%) from samples attest good chemical status of water from which: $61.90 \%$ (39 samples) having under $0.3 \mathrm{mg} / \mathrm{dm}^{3}, 14.29 \%$ (9 samples) between $0.3-0.5 \mathrm{mg} / \mathrm{dm}^{3}$, while, in $23.81 \%$ (15) 
Tab.1. The percentage of samples conform and non-conform at parameters: $\mathrm{NO}_{2}, \mathrm{NO}_{3}$ and the report $\mathrm{NO}_{3} / \mathrm{NO}_{2}$

\begin{tabular}{|c|c|c|c|c|c|c|c|c|c|c|c|c|}
\hline \multirow[t]{2}{*}{ 鸹 } & \multicolumn{2}{|c|}{$\begin{array}{c}\mathrm{KWt} \\
\mathrm{NO}_{2} \mathrm{mg} / \mathrm{dm}^{3}\end{array}$} & \multicolumn{3}{|c|}{$\begin{array}{l}\text { Fisher's test. Value of } \mathrm{NO}_{2} \\
\text { no. and } \% \text { of samples for } \\
\text { different range }\left(\mathrm{mg} / \mathrm{dm}^{3}\right)\end{array}$} & \multirow[t]{2}{*}{$\stackrel{\grave{\Xi}}{\Xi}$} & \multirow{2}{*}{$\begin{array}{c}\mathrm{KWt} \mathrm{NO} \\
\mathrm{mg} / \mathrm{dm}^{3}\end{array}$} & \multicolumn{3}{|c|}{$\begin{array}{l}\text { Fisher's test. Value } \\
\text { of } \mathrm{NO}_{3} \text {, no. and \% of } \\
\text { samples for different } \\
\text { range }\left(\mathrm{mg} / \mathrm{dm}^{3}\right)\end{array}$} & \multicolumn{2}{|c|}{$\begin{array}{c}\frac{N O 3}{50}+\frac{N O 2}{3} \leq 1 \\
\text { L.458/2002 } \\
\text { Drinking water law }\end{array}$} \\
\hline & $\begin{array}{l}\text { No.S. } \\
(\%)\end{array}$ & $\begin{array}{r} \\
X \\
\pm \text { sem } \\
\end{array}$ & $\begin{array}{c}\text { Under } \\
0.3 \\
\end{array}$ & $\begin{array}{c}\text { Between } \\
0.3-0.5 \\
\end{array}$ & $\begin{array}{c}\text { Up to } \\
0.5 \\
\end{array}$ & & & $\begin{array}{c}\text { Under } \\
50 \\
\end{array}$ & $\begin{array}{c}\text { Up to } \\
50 \\
\end{array}$ & $\begin{array}{c}\mathrm{P} \\
\text { value } \\
\end{array}$ & $\begin{array}{c}\mathrm{KWt} \\
\mathrm{X} \pm \text { sem }\end{array}$ & $\begin{array}{c}\text { No.S, limits } \\
\text { exceed } \leq 1\end{array}$ \\
\hline $\mathrm{AB}$ & 711.11 & $\begin{array}{r}0.32 \\
\pm 0.08 \\
\end{array}$ & $\begin{array}{c}4 \\
57 \% \\
\end{array}$ & $\begin{array}{c}2 \\
29 \% \\
\end{array}$ & $\begin{array}{c}1 \\
14 \% \\
\end{array}$ & $\mathrm{AB}$ & $\begin{array}{l}35.71 \\
\pm 7.53 \\
\end{array}$ & $\begin{array}{c}6 \\
86 \% \\
\end{array}$ & $\begin{array}{c}1 \\
14 \% \\
\end{array}$ & $\begin{array}{c}0.14 \\
\text { ns }\end{array}$ & $\begin{array}{c}0.82 \\
\pm 0.14 \\
\end{array}$ & $\begin{array}{c}1 \\
14 \% \\
\end{array}$ \\
\hline $\mathrm{BZ}$ & $\begin{array}{c}6 \\
9.52 \\
\end{array}$ & $\begin{array}{c}0.23 \\
\pm 0.08 \\
\end{array}$ & $\begin{array}{c}5 \\
83.83 \% \\
\end{array}$ & $\begin{array}{c}0 \\
0 \% \\
\end{array}$ & $\begin{array}{c}1 \\
16.66 \% \\
\end{array}$ & $\mathrm{BZ}$ & $\begin{array}{r}48.66 \\
\pm 5.92 \\
\end{array}$ & $\begin{array}{c}3 \\
50 \% \\
\end{array}$ & $\begin{array}{c}3 \\
50 \% \\
\end{array}$ & $\begin{array}{c}0.10 \\
\text { ns }\end{array}$ & $\begin{array}{r}1.00 \\
\pm 0.11 \\
\end{array}$ & $\begin{array}{c}3 \\
50 \% \\
\end{array}$ \\
\hline CJ & $\begin{array}{c}33 \\
52.38 \\
\end{array}$ & $\begin{array}{c}0.33 \\
\pm 0.05\end{array}$ & $\begin{array}{c}16 \\
48.48 \%\end{array}$ & $\begin{array}{c}4 \\
12.12 \% \\
\end{array}$ & $\begin{array}{c}13 \\
39.39 \%\end{array}$ & CJ & $\begin{array}{l}23.54 \\
\pm 2.82 \\
\end{array}$ & $\begin{array}{c}30 \\
91 \%\end{array}$ & $\begin{array}{c}3 \\
9 \% \\
\end{array}$ & $\begin{array}{c}0.002 \\
\text { es }\end{array}$ & $\begin{array}{c}0.55 \\
\pm 0.06 \\
\end{array}$ & $\begin{array}{c}3 \\
9 \% \\
\end{array}$ \\
\hline MS & $\begin{array}{c}11 \\
17.46 \\
\end{array}$ & $\begin{array}{r}0.23 \\
\pm 0.03 \\
\end{array}$ & $\begin{array}{c}8 \\
72.72 \% \\
\end{array}$ & $\begin{array}{c}3 \\
27.27 \% \\
\end{array}$ & $\begin{array}{c}0 \\
0 \% \\
\end{array}$ & MS & $\begin{array}{r}19.63 \\
\pm 4.64 \\
\end{array}$ & $\begin{array}{c}10 \\
91 \% \\
\end{array}$ & $\begin{array}{c}1 \\
9 \% \\
\end{array}$ & $\begin{array}{l}0.09 \\
\text { nqs }\end{array}$ & $\begin{array}{c}0.41 \\
\pm 0.09 \\
\end{array}$ & $\begin{array}{c}1 \\
9 \% \\
\end{array}$ \\
\hline SJ & $\begin{array}{r}6 \\
9.52 \\
\end{array}$ & $\begin{array}{r}0.09 \\
\pm 0.02 \\
\end{array}$ & $\begin{array}{c}6 \\
100 \% \\
\end{array}$ & $\begin{array}{c}0 \\
0 \% \\
\end{array}$ & $\begin{array}{c}0 \\
0 \% \\
\end{array}$ & SJ & $\begin{array}{l}31.16 \\
\pm 5.12 \\
\end{array}$ & $\begin{array}{c}6 \\
100 \% \\
\end{array}$ & - & - & $\begin{array}{c}0.65 \\
\pm 0.09 \\
\end{array}$ & $\begin{array}{c}0 \\
0 \% \\
\end{array}$ \\
\hline $\mathrm{T}$. & 63100 & & $\begin{array}{c}39 \\
61.90 \% \\
\end{array}$ & $\begin{array}{c}9 \\
14.29 \% \\
\end{array}$ & $\begin{array}{c}15 \\
23.81 \% \\
\end{array}$ & & & $\begin{array}{c}55 \\
87 \% \\
\end{array}$ & $\begin{array}{c}8 \\
13 \% \\
\end{array}$ & - & & \\
\hline & $\begin{array}{l}N \mathrm{t}, \mathrm{KW}= \\
=0.546 \mathrm{C}\end{array}$ & $\begin{array}{l}3.065 \\
, \mathrm{~ns} \\
\end{array}$ & $\begin{array}{l}\mathrm{F} \\
\mathrm{P}\end{array}$ & $\begin{array}{l}\text { Fisher's tes } \\
<0.0001\end{array}$ & $\begin{array}{l}\text { st } \\
\text { es. }\end{array}$ & & & $\begin{array}{r}\mathrm{Fi} \\
\mathrm{P}<\end{array}$ & $\begin{array}{l}\text { sher's t } \\
0.0001\end{array}$ & $\begin{array}{l}\text { est } \\
\text { es. }\end{array}$ & $\mathrm{P}$ & $\begin{array}{l}\text { KWt, } \\
0.054 \text {,vs }\end{array}$ \\
\hline
\end{tabular}

the aquifer is considered to be polluted: $0.55-0.91$ $\mathrm{mg} / \mathrm{dm}^{3}$ (table 1). The mean values/ counties fall in standards for drinking water, but, individual values designate poor chemical status as follows: AB: 4 samples taken from the same number of wells (0.65-0.8 mg/dm ${ }^{3}$ ), BZ: 1 sample: $0.55 \mathrm{mg} / \mathrm{dm}^{3}$; CJ: 8 samples: $0.63-0.91 \mathrm{mg} / \mathrm{dm}^{3}$. In MS the chemical status is good: $0.1-0.45 \mathrm{mg} / \mathrm{dm}^{3}$ for all samples; the same situation was found in SJ: $0.1-0.31 \mathrm{mg} /$ $\mathrm{dm}^{3}$. Concerning the "guide for completing the inspection notes concerning animal protection and welfare"; for 3 point, the limits have to be 0.3 $\mathrm{mg} / \mathrm{dm}^{3}$, therefore, $38.10 \%$ (24 samples) indicates poor water quality. Although the mean/county does not indicate nonconformities from point of view of the chemical status of water in relation to the parameter $\mathrm{NO}_{3}$, are found significant differences $\left({ }^{*} \mathrm{P}<0.05\right)$ between the mean of the analyzed samples from BZ $\left(48.66 \pm 5.92 \mathrm{mg} / \mathrm{dm}^{3}\right.$ : min. value - 35.00 - max. value: $73.00 \mathrm{mg} / \mathrm{dm}^{3}$ ) compared with those from CJ $(23.54 \pm 2.82 \mathrm{mg} /$ $\mathrm{dm}^{3}$ : min. value -8.00 - max. value: $66 \mathrm{mg} / \mathrm{dm}^{3}$ ) and MS $\left(19.63 \pm 4.64 \mathrm{mg} / \mathrm{dm}^{3}\right.$ : min. value $-10.00-$ max. value: $33.00 \mathrm{mg} / \mathrm{dm}^{3}$ ).

There is a sinuous curve of individual values, poor chemical status in terms of this parameter were recorded at one wells in $A B\left(73 \mathrm{mg} / \mathrm{dm}^{3}\right)$,
3 wells (50\%) BZ $\left(53-73 \mathrm{mg} / \mathrm{dm}^{3}\right)$, $9 \%$ from wells (3) in CJ (58-66 mg/dm $\left.{ }^{3}\right), 1$ wells (9\%) in MS $\left(63 \mathrm{mg} / \mathrm{dm}^{3}\right)$. In SJ none of the samples not present overrun at this parameter. In terms of report $\frac{\mathrm{NO} 3}{\mathrm{No}}+\frac{\mathrm{N} 2}{3} 1$ were found areas where the aquifer, is polluted. One samples from $\mathrm{AB}$ exceeds the limit: 1.55; nonconformities are registered in $\mathrm{BZ}$ at $50 \%$ from samples. In CJ 3 samples (9\%) are considered to have poor chemical status. In SJ were not found exceeding compared to the reference limit. To groundwater level, in 2013, 2014 most exceedances of the thresholds were found at $\mathrm{NO}_{3}$ parameter (Moldovan et al., 2014, Bogzianu, 2015).

\section{CONCLUSION}

In terms of nutrient regime, the overruns are influenced by poor cleaning, hygienisation and disinfection of wells.

\section{REFERENCES}

1. Bogzianu R (2015). Synthesis of water quality in Romania in 2014, www.rowater.ro

2. Grant RJ (1993). „G93-1138 Water Quality and Requirements for Dairy Cattle" Historical Materials from University of Nebraska- Lincoln Extension.Paper 445, www.digitalcommons.unl.edu 
3. Harty A (2012). Nitrates and Livestock Water Quality. http://igrow.org

4. Iacob O, Tudor A, Neamțu A, Cristea A (2012). Well water: contamination by nitrates and methemoglobinemia. Ghide for family physicians, DSP doctors and hygienists, and population. Edition I. Publisher university "Carol Davila" București
5. Moldovan C, Soare F, Dumitrache F, Iliescu S, Gheorghiu I, Rotaru N, Costea F, Nistor C (2014). Synthesis of water quality in Romania in 2013. Water resources potential and technical uses for 2013: 1-429, www.rowater.ro

6. Law No 458/ 2002 regarding the drinking water quality, published in the Official Monitor, Part I.552 din 29/07/2002 